OPEN ACCESS

Edited by:

Shibiao Wan,

St. Jude Children's Research

Hospital, United States

Reviewed by:

Tina Bianco-Miotto,

University of Adelaide, Australia

Andrew Lai,

Centre for Clinical Research

University of Queensland, Australia

*Correspondence:

Xi-rong Xiao

xiaoxirong@fudan.edu.cn

Jie-ru Zhou

doctorweny@sina.com

${ }^{\dagger}$ These authors have contributed equally to this work

Specialty section:

This article was submitted to

Computational Genomics,

a section of the journal

Frontiers in Genetics

Received: 09 July 2020

Accepted: 30 November 2020

Published: 08 January 2021

Citation:

Wang $X$, Zhu H, Lei L, Zhang $Y$, Tang C, WU J-X, Zhou J-r and Xiao X-r (2021) Integrated Analysis of Key Genes and Pathways Involved in Fetal

Growth Restriction and Their Associations With the Dysregulation of the Maternal Immune System.

Front. Genet. 11:581789.

doi: 10.3389/fgene.2020.581789

\section{Integrated Analysis of Key Genes and Pathways Involved in Fetal Growth Restriction and Their Associations With the Dysregulation of the Maternal Immune System}

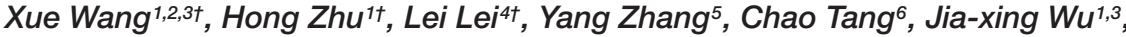 \\ Jie-ru Zhou ${ }^{1 *}$ and Xi-rong Xiao ${ }^{5 *}$ \\ ${ }^{1}$ Department of Obstetrics and Gynecology, Xin Hua Hospital Affiliated to Shanghai Jiao Tong University School of Medicine, \\ Shanghai, China, ${ }^{2}$ Ministry of Education-Shanghai Key Laboratory of Children's Environmental Health, Xin Hua Hospital \\ Affiliated to Shanghai Jiao Tong University School of Medicine, Shanghai, China, ${ }^{3}$ Shanghai Jiao Tong University School \\ of Medicine, Shanghai, China, ${ }^{4}$ Department of Obstetrics and Gynecology, East Hospital, Tongji University School \\ of Medicine, Shanghai, China, ${ }^{5}$ Obstetrics and Gynecology Hospital, Fudan University, Shanghai, China, ${ }^{6}$ Department \\ of Pharmacology, Zhejiang University Medical School, Hangzhou, China
}

Fetal growth restriction (FGR) is a common pregnancy complication and a risk factor for infant death. Most patients with FGR have preeclampsia, gestational diabetes mellitus, or other etiologies, making it difficult to determine the specific molecular mechanisms underlying FGR. In this study, an integrated analysis was performed using gene expression profiles obtained from Gene Expression Omnibus. Differentially expressed genes (DEGs) between healthy and FGR groups were screened and evaluated by functional enrichment and network analyses. In total, 80 common DEGs (FDR < 0.05) and 17 significant DEGs (FDR < 0.005) were screened. These genes were enriched for functions in immune system dysregulation in the placenta based on Gene Ontology and Kyoto Encyclopedia of Genes and Genomes pathway analyses. Among hub genes identified as candidates for FGR and fetal reprogramming, $L E P, G B P 5, H L A-D Q A 1$, and CTGF were checked by quantitative polymerase chain reaction, immunohistochemistry, and western blot assays in placental tissues. Immune imbalance could cause hypoxia environment in placenta tissues, thus regulating the fetal-reprogramming. A significant association between CTGF and HIF-1 $\alpha$ levels was confirmed in placenta tissues and HTR8 cells under hypoxia. Our results suggest that an immune imbalance in the placenta causes FGR without other complications. We provide the first evidence for roles of CTGF in FGR and show that CTGF may function via HIF-1 $\alpha$-related pathways. Our findings elucidate the pathogenesis of FGR and provide new therapeutic targets.

Keywords: fetal growth restriction, placenta, immune imbalance, fetal-reprogramming, integration analysis 


\section{INTRODUCTION}

Fetal growth restriction (FGR) is a common pregnancy complication characterized by the failure of the fetus to reach its optimal growth potential. FGR is associated with infant morbidity and mortality (Garite et al., 2004) as well as general developmental delay in both intellectual and physical (Chen et al., 2016). Although fetuses with FGR could catch up in early life, they still have a higher risk for chronic metabolic problems as adults, including type II diabetes, insulin resistance, metabolic syndrome, and cardiovascular diseases (Gatford et al., 2010; Morrison et al., 2016; Darendeliler, 2019). A number of factors could contribute to the development of FGR, including infections, drug abuse, immune disorders, hypertension, as well as anatomical factors (Adams Waldorf and McAdams, 2013; Ankumah and Sibai, 2017; Herrera et al., 2017; Sebastiani et al., 2018). However, the exact etiology remains elusive. The roles of many transcripts and hormones in the placenta in FGR have been evaluated (Okamoto et al., 2006; Hashimoto et al., 2016; Ding and Cui, 2017). However, previous studies have included patients with multiples clinical diseases, including preeclampsia, gestational diabetes mellitus, and twin births (Deyssenroth et al., 2017; Awamleh et al., 2019; Gibbs et al., 2019; Majewska et al., 2019), and studies focusing exclusively on patients with FGR are urgently needed to provide insight into the basic pathogenesis.

The placenta plays an important role in the progression of FGR owing to its influence on fetal growth (Zhu et al., 2016). Placental dysfunction is thought to be a predominant cause of FGR, and late FGR ( $\geq 32$ weeks) presents mild hypoxia with slight deficiencies in placentation (Nardozza et al., 2017). Generally, a placenta develops in a hypoxic environment associating with vascular remodeling, metabolic changes, oxidative stress, and mitochondrial dysfunction. In early pregnancy, a hypoxic environment stimulating trophoblast cells' differentiation and vascular remodeling is essential to placentation and fetal development (Soares et al., 2017). Chronic hypoxia contributes to mitochondrial dysfunction as an underlying cause of cellular mechanisms contributing to late FGR (Song et al., 2019). What's more, hypoxia is also a consequence of placental dysfunction, and low oxygen tension in the cord blood adversely affects fetal development (Soares et al., 2017). Hypoxia-inducible protein HIF- $1 \alpha$, the central factor of the cellular response to hypoxia, is closely related to the development of FGR and fetalreprogramming (Myatt, 2006). However, the molecular pathway involving HIF-1 $\alpha$ signal in FGR placenta metabolism is not clear, which needs to be further studied and worth exploring.

In this study, significant differentially expressed genes (DEGs) related to FGR were identified by transcriptome-level analyses of placental tissues. Publicly available message RNA (mRNA) profiles were downloaded from Gene Expression Omnibus (GEO) and divided into an FGR group and a healthy control group for the identification of DEGs. Significant DEGs obtained from multiple GEO datasets were further verified by quantitative polymerase chain reaction (qPCR), western blot, and immunohistochemistry (IHC) using placental tissues. Additionally, hypoxia directly or indirectly regulates several genes involved in cell differentiation; correlation analyses were performed for HIF-1 $\alpha$ and DEGs to reveal undiscovered gene regulations for interpreting links between the hypoxic environment and fetal development.

\section{MATERIALS AND METHODS}

\section{Data Collection, Processing and Differentially Expressed Gene Screening}

For mRNA profiles for placentas in six datasets were downloaded from the GEO repository. GSE98224, GSE75010, GSE100415, and GSE24129 were generated using the same platform, the GPL6244 [HuGene-1_0-st] Affymetrix Human Gene 1.0 ST Array [transcript (gene) version]. GSE10588 and GSE30186 were obtained using GPL2986 ABI Human Genome Survey Microarray Version 2, and GPL10558 was obtained using the Illumina HumanHT-12 V4.0 Expression BeadChip. To minimize interference from multiple conditions, mRNA profiles with descriptions including preeclampsia, large for gestational age, and preterm were excluded. Selected profiles were collected as GSM cohort and assigned to a healthy group or FGR group for subsequent analyses. The clinical characteristics of the GSM cohort are listed in the Supplementary Materials (GSM_profile_information.xls). GSM number is a data number for mRNA array data.

Background correction and quantile normalization of raw data were performed using the SVA package in $R$ (version 3.6.1) (Johnson et al., 2007). The limma package in $R$ was utilized to screen DEGs (Ritchie et al., 2015). ${ }^{1}$ Probes were annotated and an approximately normal distribution was obtained by $\log 2$ transformation. Differentially expressed genes screened with the thresholds FDR $<0.05$ and $\mid \log _{2}$ (Fold Change) $\mid>0.5$ were defined as common DEGs, while thresholds for significant DEGs were FDR $<0.005$ and $\mid \log _{2}$ (Fold Change) $\mid>0.7$. A heatmap and volcano plot were drawn using $R$.

\section{Bioinformatics Analysis}

A Kyoto Encyclopedia of Genes and Genomes (KEGG) pathway enrichment analysis of common DEGs was performed to discover pathways that were closely associated with FGR using the "GeneAnswers" package. ${ }^{2}$ A Gene Ontology (GO) enrichment analysis was performed using the "GOstats" package ${ }^{3}$ to evaluate the functional and biological significance of DEGs. Proteinprotein interaction (PPI) network information for common DEGs was obtained using the Search Tool for the Retrieval of Interacting Genes (STRING). ${ }^{4}$ A weighted gene co-expression network analysis (WGCNA) was performed using the WGCNA R package (Langfelder and Horvath, 2008), and networks and modules for the top $15 \%$ of genes with the largest standard deviations were obtained and visualized. Soft power thresholds (cutoff of powers $=4$, scale-free $R^{2}=0.86$ ) were used to generate modules. The PPI network and WGCNA results were visualized

\footnotetext{
${ }^{1}$ https://bioconductor.org/packages/release/bioc/html/limma.html

${ }^{2} \mathrm{http}: / /$ www.bioconductor.org/packages/release/bioc/html/GeneAnswers.html ${ }^{3} \mathrm{http}: / /$ www.bioconductor.org/packages/release/bioc/html/GOstats.html ${ }^{4} \mathrm{http}: / /$ www.string-db.org/
} 
using Cytoscape (version 3.5.1), and a value of $P<0.05$ was considered statistically significant referring to the node number.

\section{Study Population}

Twenty-seven patients with FGR (without other pregnancy diseases) and 40 healthy pregnant women were recruited from January 2018 to December 2019 at the Obstetrics and Gynecology Hospital of Fudan University and Xin Hua Hospital Affiliated to Shanghai Jiao Tong University School of Medicine, Shanghai, China. The study was approved by the Ethics Committee of the hospital (approval number: XHEC-D-2020-116). All recruited patients provided signed, informed consent prior to the collection of placental tissues. Initial recruitment for participates was performed on pregnant women at gestational age about 28 weeks, and cases of FGR were primarily screened using fetoplacental Doppler. With the progress of the pregnancy, some FGR cases were relieved. Finally, only the newborn has FGR at delivery donated their placental tissues. Thus placenta donors recruited in our study also belong to SGA. Donors met the following criteria: (1) 20-35 years of age and a gestational age of not less than 37 weeks; (2) singleton pregnancy; (3) non-smoking and nondrinking; and (4) no other pregnancy complications or genetic deficiency now or previously.

\section{Sample Processing}

The maternal decidua and fetal chorion on the surface of the placenta were removed and discarded before sampling. Due to the complex and heterogeneous characteristics of the placenta, small pieces of tissues were cut from six different regions of the placenta to avoid the impact of the spatial discrepancy, and each region was $4-6 \mathrm{~cm}$ away from the terminal of the umbilical cord. Tissue samples were taken from placentas within $30 \mathrm{~min}$ after delivery, fixed in formalin for hematoxylin-eosin (HE) staining and IHC, or frozen immediately in liquid nitrogen and stored at $-80^{\circ} \mathrm{C}$ for RNA extraction. Total RNAs were isolated from placental tissues using an RNeasy Plus Universal Kit (Cat. No. 74134; Qiagen, Hilden, Germany) according to the manufacturer's instructions. RNA quantity and quality were assessed using an ultraviolet spectrophotometer (ND1000; NanoDrop Technologies, Wilmington, DE, United States) and an electrophoresis device (Tanon 3500; Tanon, Shanghai, China), respectively.

\section{Quantitative Polymerase Chain Reaction}

Total RNA was reverse transcribed using a PrimeScript ${ }^{\mathrm{TM}}$ RT Reagent Kit containing a gDNA eraser (RR047A; Takara, Kusatsu, Japan) according to the manufacturer's instructions. Briefly, $1 \mu \mathrm{g}$ total RNA of each sample was reverse transcribed, and cDNA samples were checked about concentrations and pre-diluted to a concentration of about $100 \mathrm{ng} / \mu \mathrm{l}$ for further qPCR assay. The qPCR analysis was performed by following the MIQE guidelines (Bustin et al., 2009). Gene expression levels were detected by qPCR using the SYBR-Green PCR Kit (FP205; Tiangen, Beijing, China) and an Applied Biosystems Q7 Real-time PCR instrument (Applied Biosystems, Carlsbad, CA, United States). PCR was conducted under the following cycling conditions: $95^{\circ} \mathrm{C}$ for $15 \mathrm{~min}$ for activation, followed by 40 cycles of $95^{\circ} \mathrm{C}$ for $10 \mathrm{~s}, 58^{\circ} \mathrm{C}$ for $30 \mathrm{~s}$, and $72^{\circ} \mathrm{C}$ for $30 \mathrm{~s}$. The relative gene expression levels of candidate genes were normalized to the level of GAPDH and calculated by the comparative CT $\left(2^{-\Delta \Delta C t}\right)$ method. The sequences of primers are presented in Supplementary Table 1 . The amplification efficiencies of the genes ranged from 90 to $115 \%$. The melting curves of tested genes indicated that the amplification was specific (Supplementary Figure 1). Also, genes showed a consistent expression level using the reference gene of either GAPDH or Beta-action (Figure 4C and Supplementary Figure 2).

\section{Hematoxylin-Eosin Staining and Immunohistochemistry of Placental Tissues}

Placentas maintained in formalin were paraffin-embedded and sliced, followed by histological grading by HE staining. IHC was also performed on sections to verify the results for genes at the protein level, as described by Magaki et al. (2019), with a few modifications. Briefly, After blocking with $10 \%$ nonimmune serum, the sections were incubated with antibody to GBP5 (13220-1-AP; Proteintech, Chicago, IL, United States; 1:150 dilution), HLA-DQA1 (16918-1-AP; Proteintech; 1:100 dilution), and CTGF (23936-1-AP; Proteintech; 1:200 dilution) at room temperature for $2 \mathrm{~h}$, followed by incubation with the secondary antibody (Santa Cruz Biotechnology, United States) for $1 \mathrm{~h}$. After incubation in a streptavidin-peroxidase conjugate, the antibody complexes were visualized with diaminobenzidine tetrahydrochloride chromogen for $10 \mathrm{~s}$. Stained tissue sections were observed using an optical microscope (Olympus ${ }^{\mathrm{TM}}$ BX50, Tokyo, Japan).

\section{Cell Culture and Hypoxia Treatment}

The trophoblast cell line HTR8/SVneo (HTR8) was provided as a gift by the International Peace Maternity \& Child Health Hospital of China Welfare Institute (IPMCH). Cells were verified by STR profiling and cultured in modified RPMI1640 medium (without calcium nitrate, with L-glutamine) (Hyclone, Logan, UT, United States) supplemented with 10\% fetal bovine serum (Biowest, Nuaillé, France) and 1\% penicillin-streptomycin (Gibco, Carlsbad, CA, United States). Cells were cultured on tissue culture dishes (Thermo Fisher Scientific, Waltham, MA, United States) at $37^{\circ} \mathrm{C}$ in a $1 \% \mathrm{O}_{2}$ incubator to simulate hypoxia, while a condition of $10 \% \mathrm{O}_{2}$ has been applied for the routine culture. The HTR8 cell line was used as a model for observing the gene functions of placental extravillous trophoblast in the current study.

\section{Western Blot Analysis}

Cells cultured in a hypoxic environment were collected at 0 and $48 \mathrm{~h}$. Then, western blotting was performed to determine the expression level of CTGF. Briefly, cells were washed with cold PBS and lysed in RIPA buffer (Beyotime, Shanghai, China) on ice. Tissues were crumbled before lysing. The supernatant of each sample was collected and quantified by a commercial Bicinchoninic Acid Kit (P0012S; Beyotime Biotechnology, China) for protein determination. Then $20 \mu \mathrm{g}$ 
of protein for each sample was separated by 10\% SDS/PAGE and transferred to PVDF membranes (Roche, Basel, Switzerland). After blocking in 5\% skim milk/TBS-Tween-20 (1\%) and incubating with primary antibodies against CTGF (23936-1-AP; Proteintech; 1:1,000 dilution), HIF-1 $\alpha$ (20960-1-AP; Proteintech; 1:1,000 dilution), GAPDH (10494-1-AP; Proteintech; 1:20,000 dilution) or $\beta$-actin (20536-1-AP; Proteintech; 1:2,000 dilution), membranes were incubated with secondary antibodies and visualized using a chemiluminescence-based detection system (Pierce Biotechnology, Rockford, IL, United States). The intensity of the protein spot was analyzed using densitometry normalized to GAPDH expression.

\section{Statistical Analyses}

Data are expressed as mean values and standard deviations. The Student's $t$-test was applied to normally distributed data for comparisons between groups, and the Chi-square test was used to evaluate the sex ratio of newborns. A Pearson correlation assay was applied to the microarray data and qPCR to obtain the correlation coefficients for the relationships between the expression level of HIF- $1 \alpha$ and those of significant DEGs. A value of $P<0.05$ was considered statistically significant.

\section{RESULTS}

\section{Identification of DEGs in FGR-Related GSM Profiles}

To identify genes associated specifically with FGR without other pregnancy complications, 96 GSM profiles from six GEO datasets were downloaded, screened, and divided into a healthy group (68 healthy subjects) and an FGR group (28 FGR subjects). GSM profiles were successfully normalized (Supplementary Figure 3). A total of 80 common DEGs (FDR $<0.05)$ were screened and used to generate a heatmap (Supplementary Figure 4), and 17 genes with significant differences (FDR $<0.005)$ were considered significant DEGs (Table 1). A volcano plot and heat map of significant DEGs are shown in Figures 1A,B, respectively. A list of common DEGs is provided in the Supplementary Materials (DEGs.xls). Clinic characteristics were briefly summarized in Supplementary Table 2.

\section{Functional Enrichment Analysis of Common DEGs}

To investigate the functions and biological pathways involving common DEGs, GO enrichment, and KEGG analyses were performed. The 10 most highly significant GO terms, including biological processes, molecular functions, and cellular components, are summarized in Supplementary Table 3. As shown in Figure 2A, the common DEGs were enriched for various molecular functions (e.g., glycosaminoglycan binding), cellular components (e.g., secretory granule lumen), and biological processes (e.g., skeletal system development). The top 10 enriched KEGG pathways are summarized in Figure 2B and Supplementary Table 4. The common DEGs were mainly involved in immune and inflammatory pathways, such as Staphylococcus aureus infection, asthma, and systemic lupus erythematosus.

\section{Interactome Networks of Genes Generated by WGCNA and PPI}

To evaluate interactions among genes related to FGR, a gene co-expression network analysis using WGCNA was performed with the top $15 \%$ of genes $(1,083)$ with the largest standard deviations (Figure 3A). Five modules were extracted (shown in different colors in Figure 3). According to an analysis of module-trait relationships (Figure 3B), three modules (shown in turquoise, blue, and yellow) with $P$-values of $<0.05$ were identified as significant. Significant DEGs acting as hub genes

TABLE 1 | Significant differentially expressed genes (DEGs) in placental tissues of patients with FGR.

\begin{tabular}{|c|c|c|c|}
\hline Gene name & FDR & $\log \mathrm{FC}$ & Description \\
\hline F5 & $2.80 \mathrm{E}-12$ & -0.830 & Coagulation Factor $V$ \\
\hline PLAC1 & $6.73 E-10$ & -0.715 & Placenta Enriched 1 \\
\hline GSTA3 & $2.28 \mathrm{E}-09$ & -0.726 & Glutathione S-Transferase Alpha 3 \\
\hline TIMP1 & $2.28 \mathrm{E}-08$ & 0.740 & TIMP Metallopeptidase Inhibitor 1 \\
\hline MUC15 & 4.45E-08 & -0.770 & Mucin 15, Cell Surface Associated \\
\hline $\mathrm{CRH}$ & $1.27 \mathrm{E}-07$ & 1.143 & Corticotropin Releasing Hormone \\
\hline$A Q P 1$ & $9.50 \mathrm{E}-07$ & 0.944 & Aquaporin 1 (Colton Blood Group) \\
\hline THBS1 & 3.76E-06 & 0.777 & Thrombospondin 1 \\
\hline NNAT & $9.86 \mathrm{E}-06$ & 0.844 & Neuronatin \\
\hline$A C O X L$ & $2.81 E-05$ & -0.782 & Acyl-CoA Oxidase Like \\
\hline CTGF & $5.08 \mathrm{E}-05$ & 0.712 & Cellular Communication Network Factor 2 \\
\hline GBP5 & 0.000242 & 1.030 & Guanylate Binding Protein 5 \\
\hline FSTL3 & 0.000261 & 0.743 & Follistatin Like 3 \\
\hline LEP & 0.000338 & 1.506 & Leptin \\
\hline KCNK17 & 0.000415 & -0.830 & Potassium Two Pore Domain Channel Subfamily K Member 17 \\
\hline HLA-DQA1 & 0.000759 & 0.950 & Major Histocompatibility Complex, Class II, DQ Alpha 1 \\
\hline CXCL10 & 0.00217 & 1.319 & C-X-C Motif Chemokine Ligand 10 \\
\hline
\end{tabular}




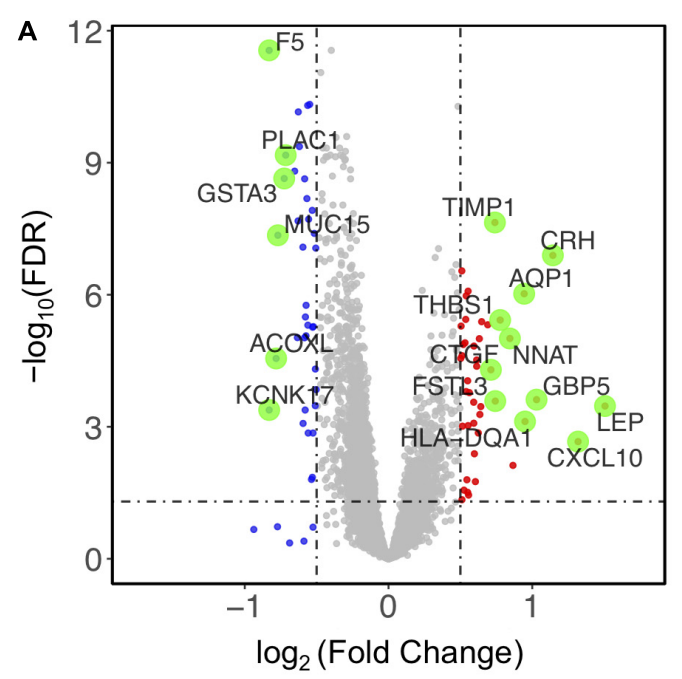

B

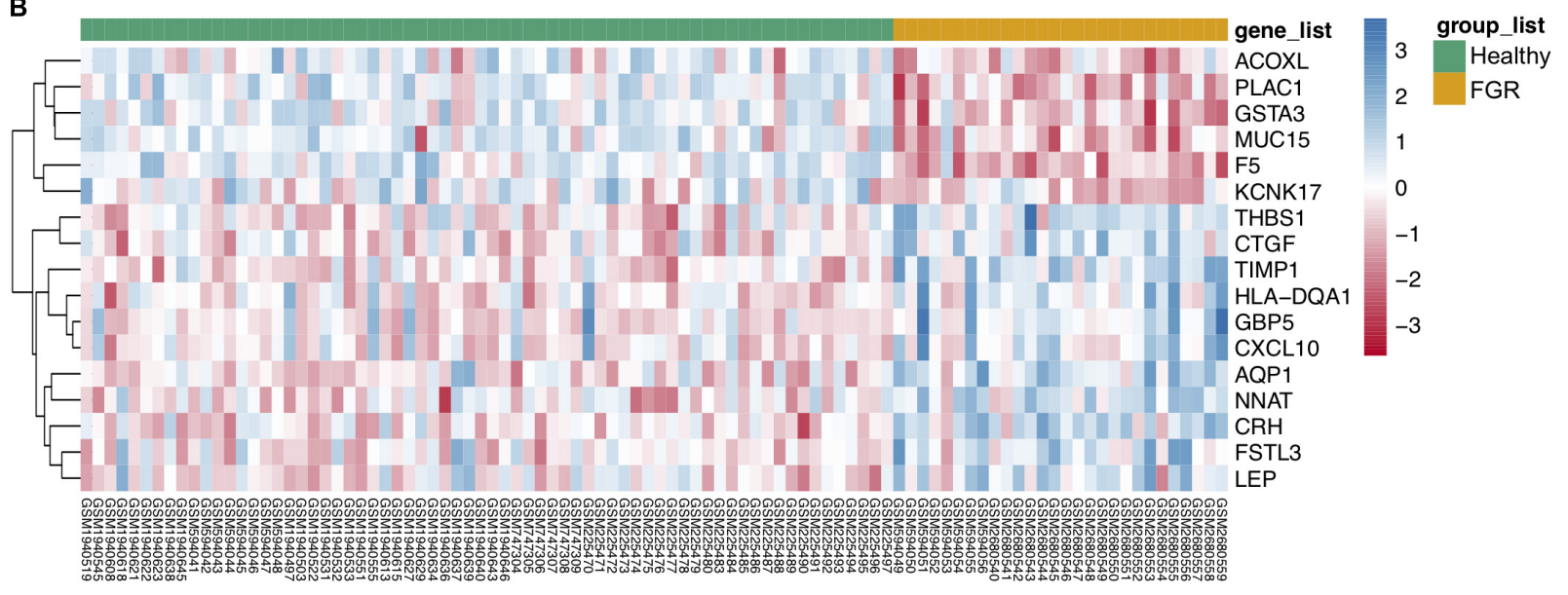

FIGURE 1 | Significant differentially expressed genes (DEGs) based on an integrated analysis. (A) Volcano map for DEGs; red dots and blue dots indicate genes that were up-regulated and down-regulated (FDR $<0.05$ ) in the fetal growth restriction (FGR) group, respectively. The green dots indicate significant DEGs (FDR $<0.005)$. (B) Two-way hierarchical clustering map of significant DEGs for FGR; upper bar in green indicates the healthy group and yellow indicates the FGR group.

in significant modules are key candidates for FGR and are listed in Table 3. The blue module shown in Figures 3C,D contained the most hub genes classified as significant DEGs. We defined the term "specific DEGs" for the significant DEGs acting as hubs in modules and networks, indicating their important roles for the pathology of FGR placentas. The module components and gene co-expression networks for the other two significantly modules (i.e., yellow and turquoise) are presented in Supplementary Figure 5.

Protein-protein interaction networks for 80 common DEGs were generated using the STRING database. TIMP1, VWF, EDN1, LEP, THBS1, CXCL10, CYR61, CTGF, ENG, and $H L A-D P A 1$ were significant $(P<0.05)$ in the PPI networks (Figure 3E). TIMP1, LEP, THBS1, CXCL10, and CTGF identified as specific DEGs are listed in Table $\mathbf{3}$ and are key genes associated with FGR.

\section{Verification of Significant DEGs in Placental Tissues}

Excluding the weight of the fetus, there were no significant differences in age, fetal gender, pregnancy age, and other characteristics between the two groups (Table 2). Quantitative PCR results suggested that GBP5 $(P=0.009$, Fold Change $=1.979), \operatorname{LEP}(P=0.002$, Fold Change $=2.359)$, HLA-DQA1 $(P=0.015$, Fold Change $=2.404)$, and CTGF $(P=0.018$, Fold Change $=0.529)$ were significantly related to FGR (Figure 4A). LEP is widely known about it relationship with FGR pathogenesis. GBP5 and HLA-DQA1 are newly defined genes associated with FGR. A previous study has reported an association between CTGF and FGR in serum and placenta (Oh et al., 2009); however, they used a very few idiopathic FGR placenta samples $(n<10)$. These results were confirmed by IHC analyses of CTGF, GBP5, and HLA-DQA1 in placental 

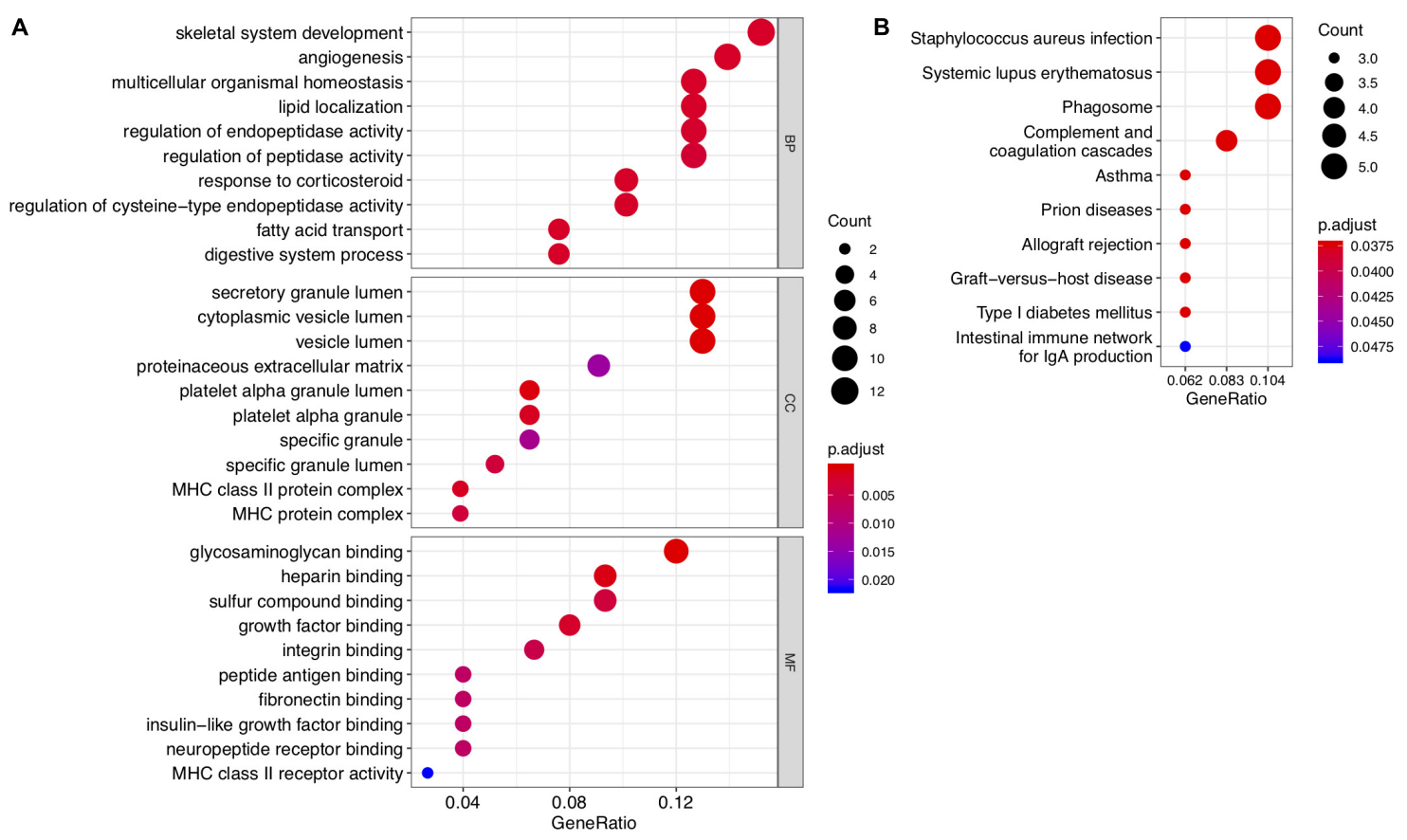

FIGURE 2 | Results of GO enrichment and KEGG pathway analyses of DEGs. (A) Top 10 enriched GO terms for common DEGs with the smallest $P$-values in the three broad categories: biological process, molecular function, and cellular component. (B) Top 10 KEGG pathways for common DEGs.

tissues (Figure 4B). Five samples in each group were used for validation. The CTGF presented a significant difference (three strong and two moderate in the FGR group, two moderate and three weak in the healthy group). The GBP presented a significant difference (two weak and three moderate in the FGR group, one moderate and four strong in the healthy group). The HLA-DQA1 presented differences but not significant (one weak, three moderate and one strong in the FGR group, three moderate and two strong in the healthy group). The western bot assay further confirmed the up-regulation of CTGF in FGR placental tissues (Figure 4C), which was consistent with the qPCR assay. Pearson correlation analysis on GEO microarray data showed a significant correlation of HIF-1 $\alpha$ and CTGF $(R=0.65, P<0.001)$, suggesting their cooperation in the pathology of placenta tissues (Figure 4D).

\section{DEGs Associated With the Hypoxic Microenvironment}

As determined by qPCR, HIF- $1 \alpha$ was significantly up-regulated in FGR placentas $(P=0.001$, Fold Change $=0.549$, Figure 5A). This result may reflect the more serious hypoxic environment for FGR placentas than for normal placentas. Pearson correlation coefficients were obtained for the relationships between mRNA levels of $H I F-1 \alpha$ and significant DEGs. CTGF was significantly

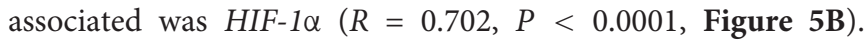
A western blot assay was conducted using the HTR8 placental trophoblast cell line to confirm the association between CTGF expression and a hypoxic environment. In HTR8 cells cultured in a hypoxic environment, CTGF expression levels were up-regulated (Figure 5C), indicating that it may be involved in $H I F-1 \alpha$-related regulatory pathways under hypoxia.

\section{DISCUSSION}

The placenta, as a source of peptides and steroid hormones, directly supports fetal development. More importantly, it provides an immune interface between the mother and the fetal allograft (Myatt, 2006). In this study, we identified 17 significant DEGs (Table 1) and 8 specific hub genes (Table 3 ) associated with FGR based on an integrated analysis of a GSM cohort. According to GO and KEGG pathway enrichment analyses (Supplementary Tables 3 and 4), the DEGs in FGR placentas were mainly enriched for functions and pathways related to an immunologic imbalance. In addition, the results for newly identified GBP5, HLA-DQA1, and CTGF were verified at the protein (IHC and western blot) and transcript (qPCR) levels, and CTGF was found as a key gene associated with idiopathic FGR. In addition, the expression of $C T G F$ is closely associated with $H I F-1 \alpha$. These results improve our understanding of the pathogenesis of FGR without other pre-gestational diseases and offer potential therapeutic targets for incurable cases. For example, therapies targeting related pathways might relieve the disorder of intracellular metabolism brought by the hypoxia environment and the immunologic imbalance, which were the two main phenotypes of the case of unexplained FGR.

Epidemiological evidence in FGR pregnancies has linked a low birth weight to fetal programming. The differential expression levels of LEP, GBP5, HLA-DQA1, and CTGF were confirmed by qPCR (Figure 4A) and these loci were identified as hub genes in 


\section{A}

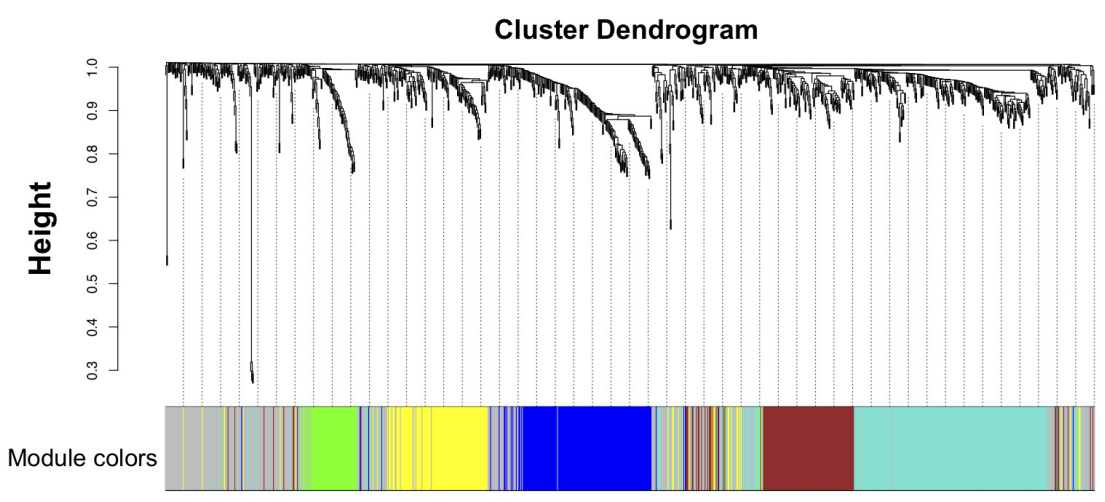

B

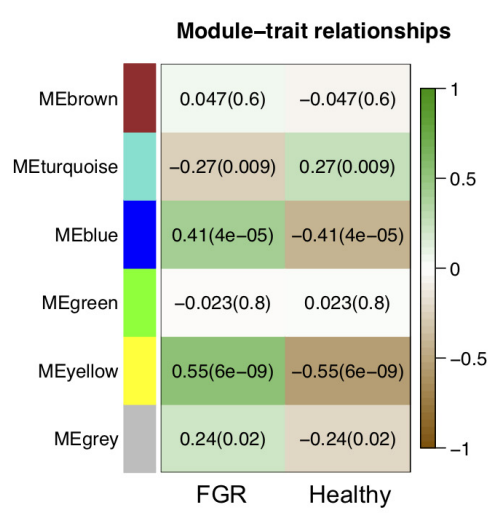

D

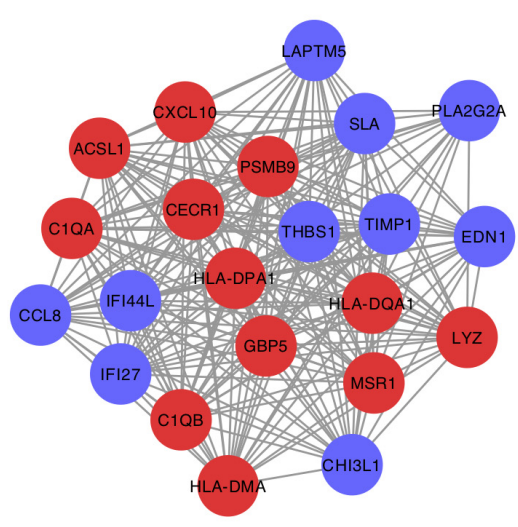

C

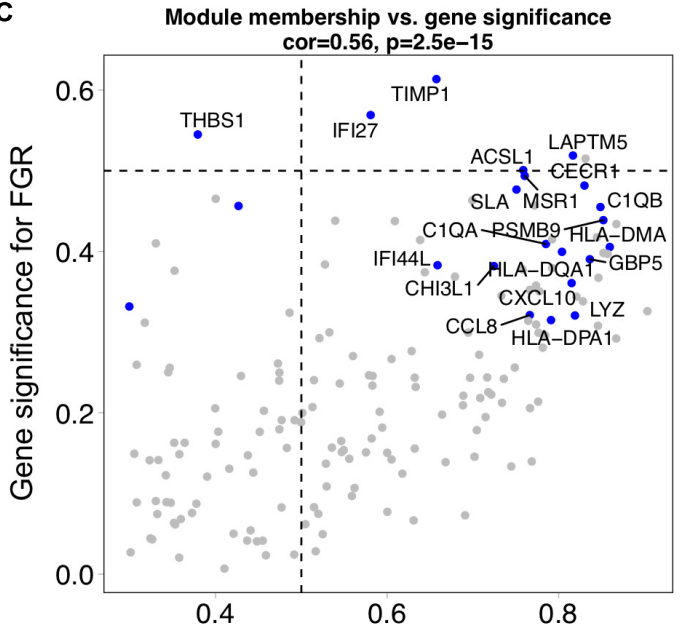

E

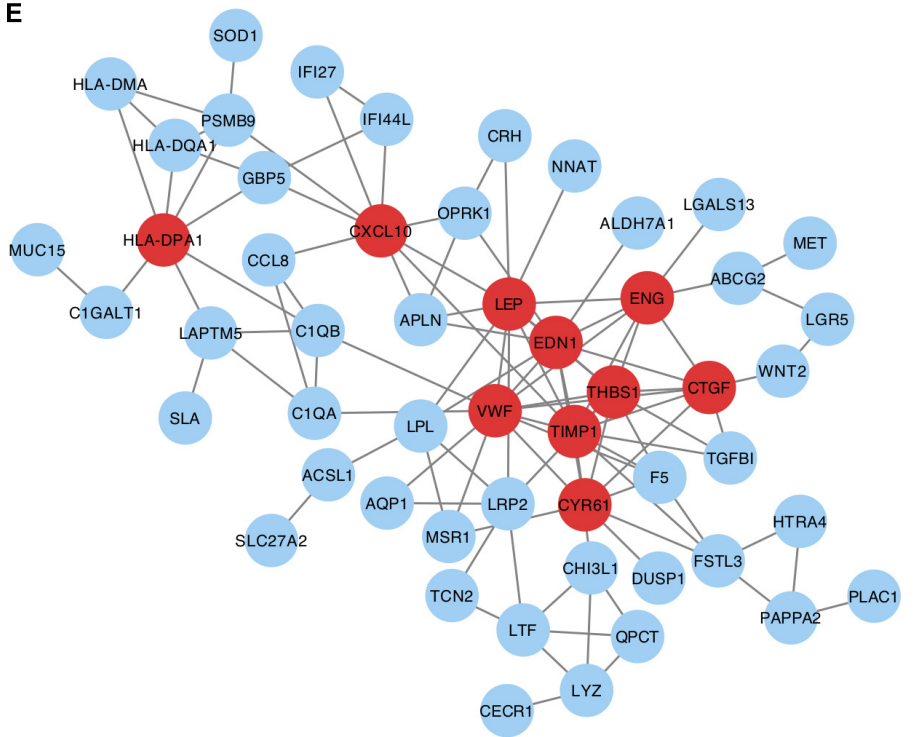

FIGURE 3 | Co-expression modules generated by weighted gene co-expression network analysis and Protein-protein interaction network analyses. (A) Cluster dendrogram of 96 samples with eligible data. Five modules (turquoise, grey, blue, brown, green, and yellow) were extracted. The grey color represents genes that cannot be classified into any module. (B) Heatmap of the correlation between DEGs in co-expression modules. (C) Scatter plot of DEGs in the blue module. (D) The blue co-expression module generated by WGCNA contained the most significant DEGs. (E) DEGs were used to generate a PPI network using the STRING online database; hub genes are indicated in red. 
TABLE 2 | Clinical characteristics of healthy individuals and patients with FGR.

\begin{tabular}{lcc}
\hline Variables & Healthy & FGR \\
\hline Age (years) & $29.13 \pm 3.39$ & $29.18 \pm 3.82$ \\
Gestational age (weeks) & $39.10 \pm 2.63$ & $38.04 \pm 2.11$ \\
Maternal ethnicity & Chinese & Chinese \\
Tissue type & Placenta & Placenta \\
Previous IUGR & No & No \\
Previous miscarriage & No & No \\
Previous hypertensive pregnancy & No & No \\
Mode proteinuria & No & No \\
HELLP diagnosis & No & Yes \\
IUGR diagnosis & No & No \\
Chorioamnionitis diagnosis & No & $11 / 16$ \\
Infant gender (Male/Female) & $22 / 18$ & $9.48 \pm 0.50$ \\
Infant weight (g) & $3,340.43 \pm 330.28$ & $2,260.00 * \pm 332.56$ \\
Aparg-score 1 min & $9.55 \pm 0.50$ & $9.48 \pm 0.50$ \\
Aparg-score 5 min & $9.88 \pm 0.33$ & $11 / 20$ \\
Mode of delivery (Cesarean & $19 / 21$ & \\
section/Eutocia) & & \\
$* P<0.01$. & &
\end{tabular}

TABLE 3 | Eight specific DEGs identified as hub genes in the WGCNA or PPI analysis were determined to be key genes for the pathogenesis of FGR.

\begin{tabular}{lccc}
\hline Gene name & qPCR-verified & WGCNA modules & PPI network \\
\hline TIMP1 & $\times$ & $\times$ & $\bigcirc$ \\
MUC15 & $\times$ & "turquoise" & $\times$ \\
THBS1 & $\times$ & $\times$ & $\bigcirc$ \\
CTGF & $\bigcirc$ & $\times$ & $\bigcirc$ \\
GBP5 & $\bigcirc$ & "blue" & $\times$ \\
LEP & $\bigcirc$ & $\times$ & $\bigcirc$ \\
HLA-DQA1 & $\bigcirc$ & "blue" & $\times$ \\
CXCL10 & $\times$ & "blue" & $\bigcirc$ \\
\hline
\end{tabular}

networks and thereby likely play key roles in FGR (Figure 3D,E and Table 3). LEP, the most highly significantly downregulated gene in FGR placentas, encodes leptin, found in the circulation. Leptin plays a major role in the regulation of energy homeostasis and obesity and is involved in endocrine functions, including the regulation of immune and inflammatory responses, angiogenesis, reproduction, and bone formation (Bodary et al., 2007; Steinbrekera and Roghair, 2016). Maternal and fetal leptin levels are correlated with FGR originating from damaged placental function (Zareaan et al., 2017). CTGF, the most significant up-regulated gene, encodes connective tissue growth factor. This factor is related to platelet-derived growth factor and plays roles in chondrocyte proliferation and differentiation as well as cell adhesion (Fujisawa et al., 2008; Kiwanuka et al., 2011). Additionally, as shown in Supplementary Table 3, CTGF contributed to the top $5 \mathrm{GO}$ terms in the molecular function category (i.e., glycosaminoglycan binding, heparin binding, growth factor binding, sulfur compound binding, and integrin binding) and the top $2 \mathrm{GO}$ terms in the biological process category (i.e., response to corticosteroid and skeletal system development), suggesting that it influences the metabolism and immune function in fetal development. Significantly downregulated GBP5 encodes guanylate binding protein 5, which is related to the innate immune system and the NF- $\mathrm{B}$ pathway. Previous studies have suggested that GBP5 is involved in the mechanism underlying FGR caused by post-natal innate immune alterations. An altered innate immune system is associated with FGR based on the increased susceptibility to infections in the postnatal period (Amdi et al., 2020). Additionally, the NF- $\kappa B$ pathway contributes to vascular growth in the developing fetal lung, and the maintenance of endothelial NF- $\kappa \mathrm{B}$ activation may be useful in FGR marked by disrupted angiogenesis (Dodson et al., 2018). The significantly down-regulated gene HLA-DQA1 encodes a membrane-anchored protein for presenting peptides derived from extracellular proteins. It is mainly expressed in lymphocytes, dendritic cells, and macrophages; these antigenpresenting cells show altered plasticity in FGR placentas (Freitag et al., 2014; Liu et al., 2015; Chu et al., 2019). In addition, HLA$D Q A 1$ was involved in 8 of the top 10 KEGG pathways (see Supplementary Table 3), indicating that it is closely associated with FGR and fetal programming via immunologic processes.

$H I F-1 \alpha$ activates gene transcription in response to changes in oxygen concentrations and accumulates in FGR placentas (Tal et al., 2010). HIF-1 $\alpha$ transcription levels were significantly upregulated in this study. A previous study has demonstrated that maternal inflammation-induced FGR is associated with increased placental HIF-1 $\alpha$ (Robb et al., 2017); thus, we hypothesize that the hypoxic microenvironment presenting in most FGR placentas (Figure 5A) could be attributed to immune dysfunction. This inference is supported by the results of our bioinformatics analyses. The hypoxic environment induced oxidative DNA damage in the placenta in FGR (Kimura et al., 2013), which may explain the link between the epidemiological characteristics of FGR and fetal reprogramming. In addition, we obtained the first evidence for a correlation between the expression levels of CTGF and HIF-1 $\alpha$. Previous reports have demonstrated that CTGF could be affected by a hypoxic environment (Rimon et al., 2008). Our western blot assay using HTR8 cells confirmed the accumulation of CTGF under hypoxia, further suggesting CTGF contributes to hypoxia-related pathways. This finding reveals a common mechanism linking the hypoxic environment to fetal programming as well as the pathogenesis of FGR.

Unlike gestational diabetes mellitus- and preeclampsiainduced FGR, which are mainly explained by metabolic syndrome or hypertensive disorder (Ornoy, 2011; Gibbs et al., 2019), our results suggested that the main cause of FGR without other complications is probably abnormal immune functions in the placenta. The novel key genes (GBP5, HLA$D Q A 1$, and $C T G F)$ provide insight into the mechanism underlying FGR development and fetal programming and provide therapeutic targets for improving prognosis. Despite the important discoveries, our study had some limitations. Research revealing biomarkers depends on population-based analyses; however, FGR placentas without other diseases are not easy to collect and thus a larger sample size could improve the discovery of specific FGR-related genes. Furthermore, experiments using cell and animal models are necessary to confirm the newly identified genes and clarify their precise roles. 


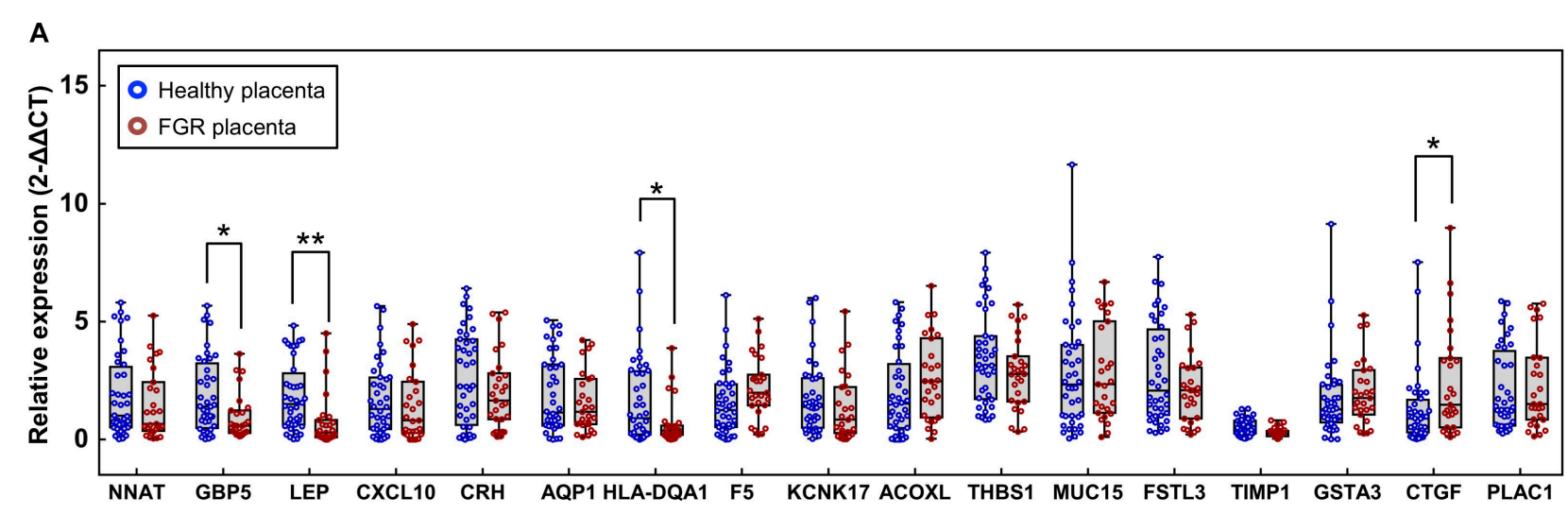

B
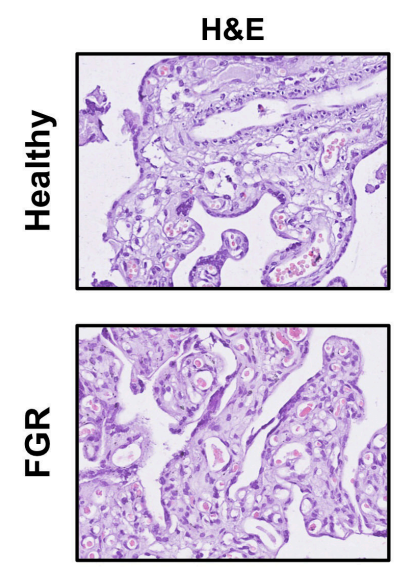
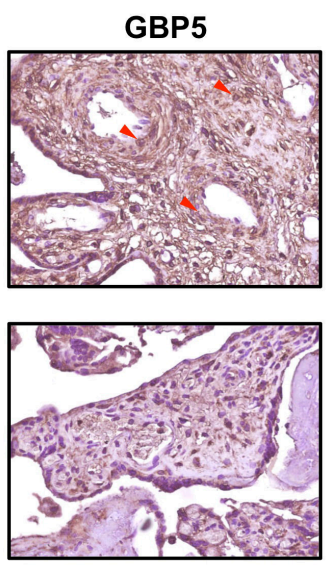

HLA- DQA1
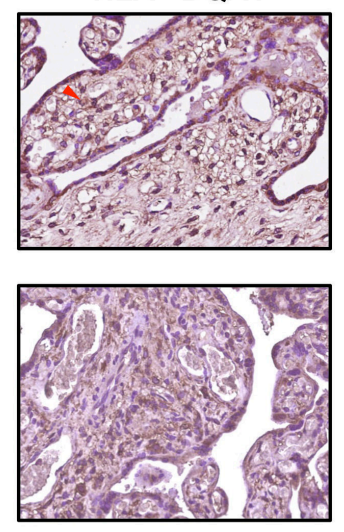
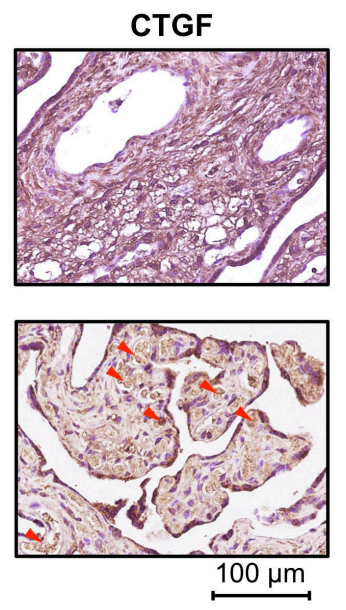

C
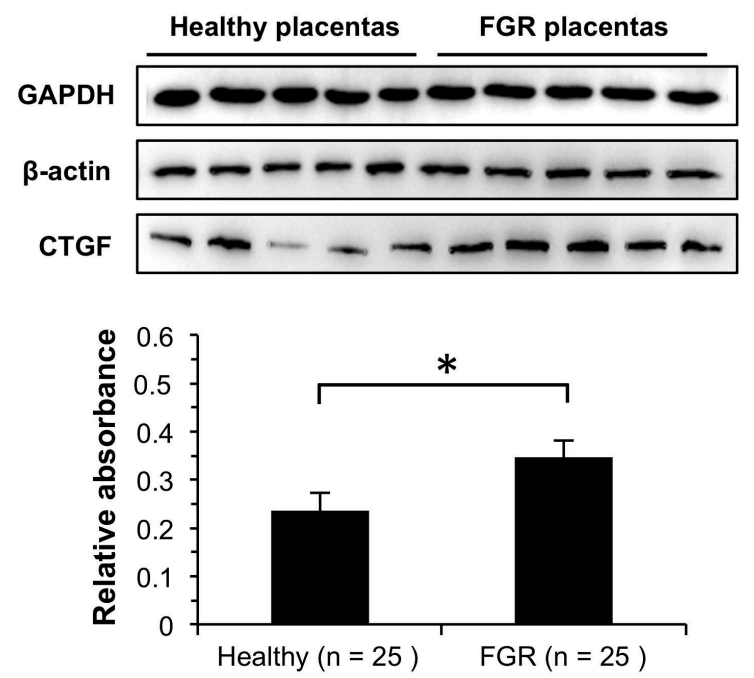

D

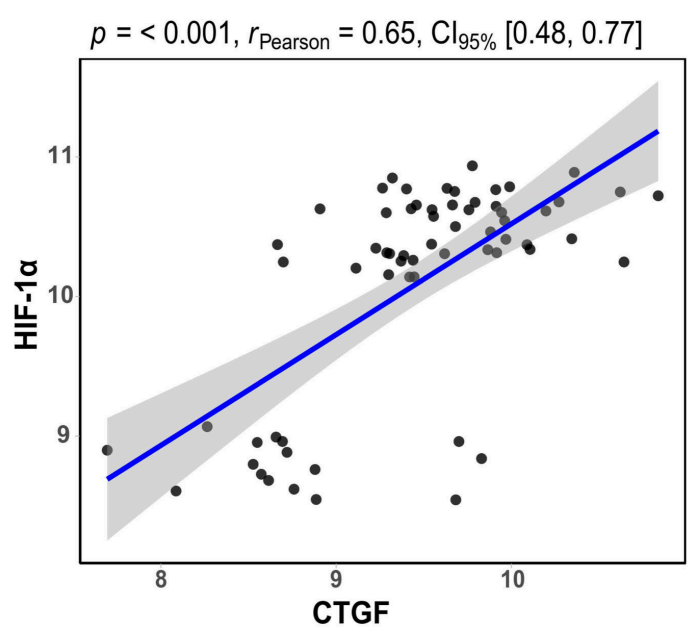

FIGURE 4 | Verification of significant DEGs at the transcript and protein levels. (A) Quantitative polymerase chain reaction (qPCR) analysis of placental tissues to verify the transcript levels of significant DEGs. (B) Representative immunohistochemistry results for placenta tissues to confirm candidate DEGs. (C) CTGF protein, quantified using western blotting. Representative five blots are shown. (D) The expression level of CTFG in GEO microarray data was significantly associated with that of HIF-1 $\alpha .{ }^{*} P<0.05,{ }^{* *} P<0.005$. 
A
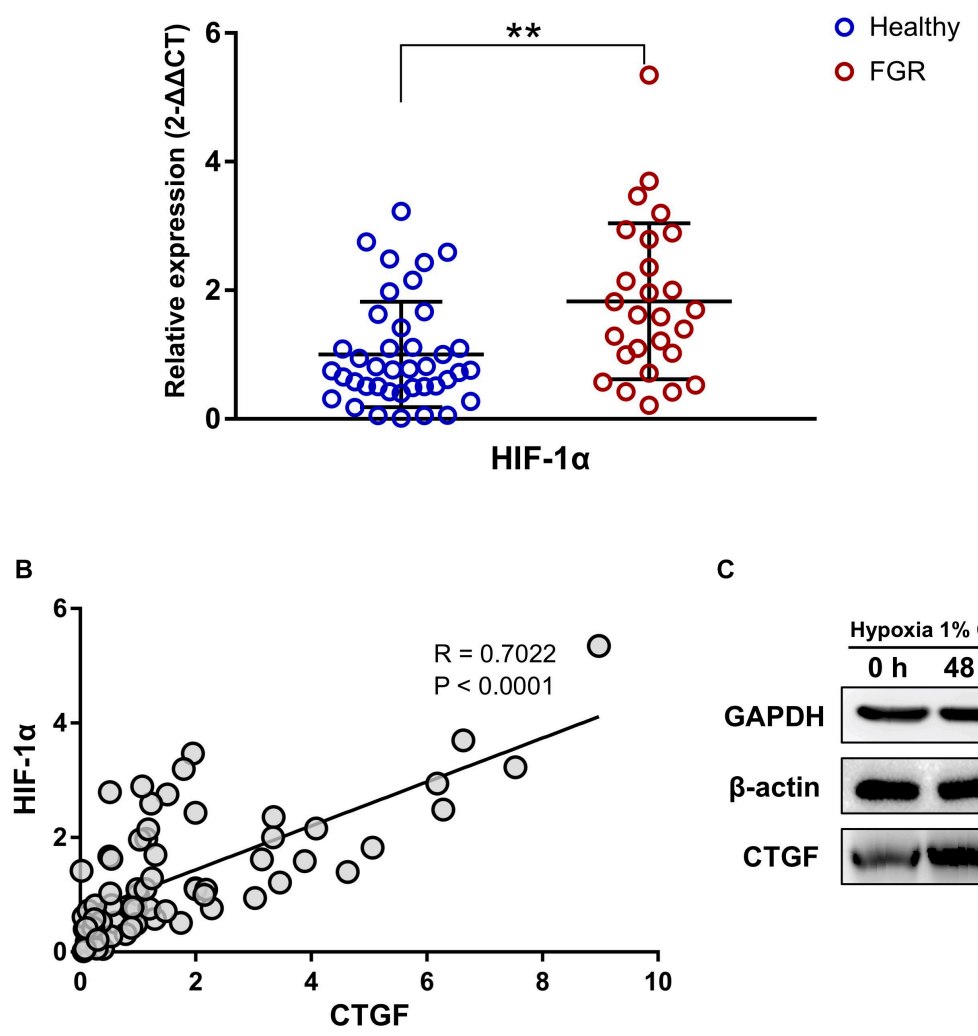

C

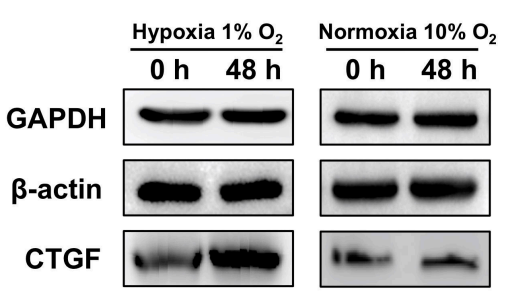

FIGURE 5 | The expression level of CTFG was related to the "hypoxic" environment and was associated with the level of HIF-1 $\alpha$. (A) The significant up-regulation of HIF-1 a was revealed by a qPCR assay of placental tissues. (B) The expression level of CTFG was significantly associated with that of $H I F-1 \alpha$. (C) Compared to the normoxic control, CTFG levels were higher in the "hypoxic" environment. ${ }^{* *} P<0.005$.

\section{DATA AVAILABILITY STATEMENT}

Publicly available datasets were analyzed in this study. This data can be found here: Six datasets were downloaded from the GEO repository: GSE98224, GSE75010, GSE100415, GSE24129, GSE10588, and GSE30186.

\section{ETHICS STATEMENT}

The studies involving human participants were reviewed and approved by The study was approved by the Ethics Committee of the hospital (approval number: XHEC-D-2020-116). The patients/participants provided their written informed consent to participate in this study.

\section{AUTHOR CONTRIBUTIONS}

XW and X-rX: conceptualization, funding acquisition, and review and editing. $\mathrm{HZ}$ and $\mathrm{YZ}$ : data curation and analysis. $\mathrm{HZ}$ and J-rZ: investigation. J-xW: resources. XW: data visualization and writing draft. J-rZ and X-rX: supervision. CT: validation. All authors contributed to the article and approved the submitted version.

\section{FUNDING}

This work was supported by the Ministry of Education, Shanghai Key Laboratory of Children's Environmental Health, Xinhua Hospital, Shanghai Jiao Tong University School of Medicine; China Youth Program of National Natural Science Foundation of China (Grant Nos. 81801543 and 81701470), National Science Foundation of China (Grant No. 81871183), and China Postdoctoral Science Foundation (Grant No. 2018M642044).

\section{ACKNOWLEDGMENTS}

Thanks to Liu Jin, our best friends, for supporting us a lot during our hard-working time.

\section{SUPPLEMENTARY MATERIAL}

The Supplementary Material for this article can be found online at: https://www.frontiersin.org/articles/10.3389/fgene.2020. 581789/full\#supplementary-material 


\section{REFERENCES}

Adams Waldorf, K. M., and McAdams, R. M. (2013). Influence of infection during pregnancy on fetal development. Reproduction 146, R151-R162. doi: 10.1530/ REP-13-0232

Amdi, C., Lynegaard, J. C., Thymann, T., and Williams, A. R. (2020). Intrauterine growth restriction in piglets alters blood cell counts and impairs cytokine responses in peripheral mononuclear cells 24 days post-partum. Sci. Rep. 10:4683. doi: 10.1038/s41598-020-61623-w

Ankumah, N. E., and Sibai, B. M. (2017). Chronic hypertension in pregnancy: diagnosis, management, and outcomes. Clin. Obstet. Gynecol. 60, 206-214. doi: 10.1097/GRF.0000000000000255

Awamleh, Z., Gloor, G. B., and Han, V. K. M. (2019). Placental microRNAs in pregnancies with early onset intrauterine growth restriction and preeclampsia: potential impact on gene expression and pathophysiology. BMC Med. Genomics 12:91. doi: 10.1186/s12920-019-0548-x

Bodary, P. F., Shen, Y., Ohman, M., Bahrou, K. L., Vargas, F. B., Cudney, S. S., et al. (2007). Leptin regulates neointima formation after arterial injury through mechanisms independent of blood pressure and the leptin receptor/STAT3 signaling pathways involved in energy balance. Arterioscler. Thromb. Vasc. Biol. 27, 70-76. doi: 10.1161/01.ATV.0000252068.89775.ee

Bustin, S. A., Benes, V., Garson, J. A., Hellemans, J., Huggett, J., Kubista, M., et al. (2009). The MIQE guidelines: minimum information for publication of quantitative real-time PCR experiments. Clin. Chem. 55, 611-622. doi: 10.1373/ clinchem.2008.112797

Chen, J., Chen, P., Bo, T., and Luo, K. (2016). Cognitive and behavioral outcomes of intrauterine growth restriction school-age children. Pediatrics 137:e20153868. doi: 10.1542/peds.2015-3868

Chu, A., Najafzadeh, P., Sullivan, P., Cone, B., Elshimali, R., Shakeri, H., et al. (2019). Aldehyde dehydrogenase isoforms and inflammatory cell populations are differentially expressed in term human placentas affected by intrauterine growth restriction. Placenta 81, 9-17. doi: 10.1016/j.placenta.2019.03.015

Darendeliler, F. (2019). IUGR: genetic influences, metabolic problems, environmental associations/triggers, current and future management. Best Pract. Res. Clin. Endocrinol. Metab. 33:101260. doi: 10.1016/j.beem.2019.01.001

Deyssenroth, M. A., Peng, S., Hao, K., Lambertini, L., Marsit, C. J., and Chen, J. (2017). Whole-transcriptome analysis delineates the human placenta gene network and its associations with fetal growth. BMC Genomics 18:520. doi: 10.1186/s12864-017-3878-0

Ding, Y. X., and Cui, H. (2017). Integrated analysis of genome-wide DNA methylation and gene expression data provide a regulatory network in intrauterine growth restriction. Life Sci. 179, 60-65. doi: 10.1016/j.lfs.2017.04. 020

Dodson, R. B., Powers, K. N., Gien, J., Rozance, P. J., Seedorf, G., Astling, D., et al. (2018). Intrauterine growth restriction decreases NF-кB signaling in fetal pulmonary artery endothelial cells of fetal sheep. Am. J. Physiol. Lung Cell. Mol. Physiol. 315, L348-L359. doi: 10.1152/ajplung.00052.2018

Freitag, N., Zwier, M. V., Barrientos, G., Tirado-González, I., Conrad, M. L., Rose, M., et al. (2014). Influence of relative NK-DC abundance on placentation and its relation to epigenetic programming in the offspring. Cell Death Dis. 5:e1392. doi: $10.1038 /$ cddis. 2014.353

Fujisawa, T., Hattori, T., Ono, M., Uehara, J., Kubota, S., Kuboki, T., et al. (2008). $\mathrm{CCN}$ family 2/connective tissue growth factor (CCN2/CTGF) stimulates proliferation and differentiation of auricular chondrocytes. Osteoarthr. Cartilage 16, 787-795. doi: 10.1016/j.joca.2007.11.001

Garite, T. J., Clark, R., and Thorp, J. A. (2004). Intrauterine growth restriction increases morbidity and mortality among premature neonates. Am. J. Obstet. Gynecol. 191, 481-487. doi: 10.1016/j.ajog.2004.01.036

Gatford, K. L., Simmons, R. A., De Blasio, M. J., Robinson, J. S., and Owens, J. A. (2010). Placental programming of postnatal diabetes and impaired insulin action after IUGR. Placenta 31, S60-S65. doi: 10.1016/j.placenta.2009.12.015

Gibbs, I., Leavey, K., Benton, S. J., Grynspan, D., Bainbridge, S. A., and Cox, B. J. (2019). Placental transcriptional and histologic subtypes of normotensive fetal growth restriction are comparable to preeclampsia. Am. J. Obstet. Gynecol. 220, 110.e1-110.e21. doi: 10.1016/j.ajog.2018.10.003

Hashimoto, Y., Kawai, M., Nagai, S., Matsukura, T., Niwa, F., Hasegawa, T., et al. (2016). Fetal growth restriction but not preterm birth is a risk factor for severe hypospadias. Pediatr. Int. 58, 573-577. doi: 10.1111/ped.12864
Herrera, E. A., Cifuentes-Zúñiga, F., Figueroa, E., Villanueva, C., Hernández, C., Alegría, R., et al. (2017). N-acetylcysteine, a glutathione precursor, reverts vascular dysfunction and endothelial epigenetic programming in intrauterine growth restricted guinea pigs. J. Physiol. 595, 1077-1092. doi: 10.1113/JP273396

Johnson, W. E., Li, C., and Rabinovic, A. (2007). Adjusting batch effects in microarray expression data using empirical Bayes methods. Biostatistics 8, 118-127. doi: 10.1093/biostatistics/kxj037

Kimura, C., Watanabe, K., Iwasaki, A., Mori, T., Matsushita, H., Shinohara, K., et al. (2013). The severity of hypoxic changes and oxidative DNA damage in the placenta of early-onset preeclamptic women and fetal growth restriction. J. Matern. Fetal Neonatal Med. 26, 491-496. doi: 10.3109/14767058.2012. 733766

Kiwanuka, E., Andersson, L., Caterson, E. J., Junker, J. P., Gerdin, B., and Eriksson, E. (2011). CCN2 promotes keratinocyte adhesion and migration via integrin

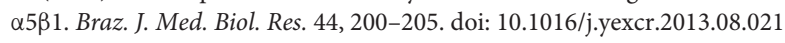

Langfelder, P., and Horvath, S. (2008). WGCNA: an R package for weighted correlation network analysis. BMC Bioinformatics 9:559. doi: 10.1186/14712105-9-559

Liu, Y., He, S., Zhang, Y., Xia, W., Li, M., Zhang, C., et al. (2015). Effects of intrauterine growth restriction during late pregnancy on the development of the ovine fetal thymus and the T-lymphocyte subpopulation. Am. J. Reprod. Immunol. 74, 26-37. doi: 10.1111/aji.12371

Magaki, S., Hojat, S. A., Wei, B., So, A., and Yong, W. H. (2019). An introduction to the performance of immunohistochemistry. Methods Mol. Biol. 1897, 289-298. doi: 10.1007/978-1-4939-8935-5_25

Majewska, M., Lipka, A., Paukszto, L., Jastrzebski, J. P., Szeszko, K., Gowkielewicz, M., et al. (2019). Placenta transcriptome profiling in intrauterine growth restriction (IUGR). Int. J. Mol. Sci. 20:1510. doi: 10.3390/ijms20061510

Morrison, K. M., Ramsingh, L., Gunn, E., Streiner, D., Van Lieshout, R., Boyle, M., et al. (2016). Cardiometabolic health in adults born premature with extremely low birth weight. Pediatrics 138:e20160515. doi: 10.1542/peds.2016-0515

Myatt, L. (2006). Placental adaptive responses and fetal programming. J. Physiol. 572(Pt 1), 25-30. doi: 10.1113/jphysiol.2006.104968

Nardozza, L. M., Caetano, A. C., Zamarian, A. C., Mazzola, J. B., Silva, C. P., Marçal, V. M., et al. (2017). Fetal growth restriction: current knowledge. Arch. Gynecol. Obstet. 295, 1061-1077. doi: 10.1007/s00404-017-4341-9

Oh, S. Y., Song, S. E., Seo, E. S., Kim, K. H., Choi, S. J., Suh, Y. L., et al. (2009). The expression of connective tissue growth factor in pregnancies complicated by severe preeclampsia or fetal growth restriction. Placenta 30, 981-987. doi: 10.1016/j.placenta.2009.08.006

Okamoto, A., Endo, H., Kalionis, B., Shinya, M., Saito, M., Nikaido, T., et al. (2006). IGFBP1 and Follistatin-like 3 genes are significantly up-regulated in expression profiles of the IUGR placenta. Placenta 27, 317-321. doi: 10.1016/j.placenta. 2004.12.007

Ornoy, A. (2011). Prenatal origin of obesity and their complications: gestational diabetes, maternal overweight and the paradoxical effects of fetal growth restriction and macrosomia. Reprod. Toxicol. 32, 205-212. doi: 10.1016/j. reprotox.2011.05.002

Rimon, E., Chen, B., Shanks, A. L., Nelson, D. M., and Sadovsky, Y. (2008). Hypoxia in human trophoblasts stimulates the expression and secretion of connective tissue growth factor. Endocrinology 149, 2952-2958. doi: 10.1210/en.20071099

Ritchie, M. E., Phipson, B., Wu, D., Hu, Y., Law, C. W., Shi, W., et al. (2015). limma powers differential expression analyses for RNA-sequencing and microarray studies. Nucleic Acids Res. 43:e47. doi: 10.1093/nar/gkv007

Robb, K. P., Cotechini, T., Allaire, C., Sperou, A., and Graham, C. H. (2017). Inflammation-induced fetal growth restriction in rats is associated with increased placental HIF-1 $\alpha$ accumulation. PLoS One 12:e0175805. doi: 10.1371/ journal.pone.0175805

Sebastiani, G., Borrás-Novell, C., Casanova, M. A., Pascual Tutusaus, M., Ferrero Martínez, S., Gómez Roig, M. D., et al. (2018). The effects of alcohol and drugs of abuse on maternal nutritional profile during pregnancy. Nutrients 10:E1008. doi: $10.3390 /$ nu10081008

Soares, M. J., Iqbal, K., and Kozai, K. (2017). Hypoxia and placental development. Birth Defects Res. 109, 1309-1329. doi: 10.1002/bdr2.1135

Song, H., Telugu, B. P., and Thompson, L. P. (2019). Sexual dimorphism of mitochondrial function in the hypoxic guinea pig placenta. Biol. Reprod. 100, 208-216. doi: 10.1093/biolre/ioy167 
Steinbrekera, B., and Roghair, R. (2016). Modeling the impact of growth and leptin deficits on the neuronal regulation of blood pressure. J. Endocrinol. 231, R47-R60. doi: 10.1530/JOE-16-0273

Tal, R., Shaish, A., Barshack, I., Polak-Charcon, S., Afek, A., Volkov, A., et al. (2010). Effects of hypoxia-inducible factor-1alpha overexpression in pregnant mice: possible implications for preeclampsia and intrauterine growth restriction. Am. J. Pathol. 177, 2950-2962. doi: 10.2353/ajpath.2010.090800

Zareaan, E., Heidarpour, M., Kargarzadeh, E., and Moshfeghi, M. (2017). Association of maternal and umbilical cord blood leptin concentrations and abnormal color Doppler indices of umbilical artery with fetal growth restriction. Int. J. Reprod. Biomed. 15, 135-140. doi: 10.29252/ijrm.15. 3.135

Zhu, M. Y., Milligan, N., Keating, S., Windrim, R., Keunen, J., Thakur, V., et al. (2016). The hemodynamics of late-onset intrauterine growth restriction by
MRI. Am. J. Obstet. Gynecol. 214, 367.e1-367.e17. doi: 10.1016/j.ajog.2015. 10.004

Conflict of Interest: The authors declare that the research was conducted in the absence of any commercial or financial relationships that could be construed as a potential conflict of interest.

Copyright (c) 2021 Wang, Zhu, Lei, Zhang, Tang, Wu, Zhou and Xiao. This is an open-access article distributed under the terms of the Creative Commons Attribution License (CC BY). The use, distribution or reproduction in other forums is permitted, provided the original author(s) and the copyright owner(s) are credited and that the original publication in this journal is cited, in accordance with accepted academic practice. No use, distribution or reproduction is permitted which does not comply with these terms. 